Yeast 2007; 24: 76I-766.

Published online 28 June 2007 in Wiley InterScience

(www.interscience.wiley.com) DOI: 10.1002/yea.15II

\title{
Yeast vectors for the integration/expression of any sequence at the TYRI locus
}

\author{
Mario G. Mirisola*, Leonarda Colomba, Alessia Gallo, Roberta Amodeo and Giacomo De Leo \\ Dipartimento di Biopatologia e Metodologie Biomediche, Università degli Studi di Palermo, Italy
}

* Correspondence to:

Mario G. Mirisola,

Via Divisi 83,

Palermo 90133

Italy.

E-mail:mirisola@unipa.it
Received: 22 January 2007

Accepted: 14 May 2007

\begin{abstract}
We have constructed new yeast vectors for targeted integration and conditional expression of any sequence at the Saccharomyces cerevisiae TYRI locus which becomes disrupted. We show that vector integration is not neutral, causing prototrophy for tyrosine and auxotrophy for the vector's selectable marker (uracil or leucine, depending on the vector used). This feature allows a double screening of transformed yeast cells, improving the identification of colonies with the desired chromosomal structure. The GAL10 gene promoter has been added to drive conditional expression of cloned sequences. Using these vectors, chromosomal structure verification of recombinant clones is no longer necessary, since the noise of non-homologous recombination, as well as spontaneous reversion of the selected phenotype, can easily be identified. The ability of the vector to conditionally control gene expression has been confirmed using the gene for the green fluorescent protein (GFP) as a reporter. GenBank Accession Nos EF202083-202086. Copyright $₫ 2007$ John Wiley \& Sons, Ltd.
\end{abstract}

Keywords: yeast integration vector; TYRI locus; expression vectors; heterologous expression; ends-out gene targeting

\section{Introduction}

The yeast Saccharomyces cerevisiae is a widely used eukaryotic model organism. It has been demonstrated to be one of the most valuable model systems for the understanding, among other things, of cell cycle control (Hartwell, 2002), protein trafficking (Bowens and Stevens, 2005; Nakano, 2004), protein folding and misfolding (Coughlan et al., 2005; Lindquist, 2002) and, more recently, ageing and neurosciences (Longo, 2004; Kaeberlein et al., 2001; Tissenbaum et al., 2002; Sherman and Muchowsky, 2003). Most genetic screens, in all these research fields, manipulate the yeast genome by removing or introducing DNA sequences. A Sequence Of Interest (SOI) may be introduced in different ways, but chromosomal gene targeting is surely one of the preferred choices. Stable integration does not need selective media and copy number cannot fluctuate either during growth or in comparing different experiments (Schneider and Guarente, 1991). In addition, stable recombinant yeast cells with different relevant genotypes may be crossed and the resulting new genetic combinations studied, e.g. for synthetic lethality. Integration of SOI is often accomplished by cloning the SOI into one of the yeast integrative plasmid (YIp) families and using a procedure known as 'ends-in' gene targeting (Langston and Symington, 2004; Hastings et al., 1993). This procedure, based on recombination between the vector selectable marker and its homologous chromosomal sequence, generates a duplication of the marker, whose wild-type copy restores the auxotrophic phenotype. Unfortunately, these repeated sequences may eject the integrated SOI by homologous intrachromosomal recombination. The uninterrupted selective pressure for the marker may help to solve the problem, but there is still the possibility of a recombination event that eliminates the 
SOI but leaves a wild-type copy of the marker. Furthermore, the chromosomal structure of the target locus must be confirmed by Southern blotting or PCR, since random integration of the SOI within the yeast genome is always a possible unintended consequence.

However, gene replacement has been improved with a procedure called 'ends-out'. This is a threestep method. First, the target sequence is cloned in a bacterial vector. Second, the main internal part of the cloned sequence is substituted by a selectable marker, generating a vector containing the selectable marker flanked on both sides by two small fragments of the target sequence. Finally, the vector is cut in order to release and purify the selectable marker flanked by at least $40-50 \mathrm{bp}$ of the target sequences. This fragment is called a recombination cassette, since it can direct the integration of the selectable marker within the chromosomal sequence homologous to the vector's target sequences. This procedure (Langston and Symington, 2004; Hastings et al., 1993) generates the integration of the marker within the chromosomal target sequence but does not create sequence duplications, and thus increases the chromosomal stability of integrants.

Yeast integrative vectors for general use have so far been based on the ends-in strategy, with all the limitations mentioned above. Voth et al. (2001) have reported the construction of some general yeast vectors capable of driving ends-out integration at the $\mathrm{HO}$ locus. Unfortunately, since these vectors lack a yeast promoter, they can only be used to integrate yeast genes along with their own promoter. In addition, integration at the $\mathrm{HO}$ locus is neutral and correct integrants must be identified by Southern blotting or PCR.

We report here the construction of new yeast vectors capable of targeting any SOI onto the TYRI locus. The integration procedure is based on the ends-out strategy and does not generate tandem duplication. In addition, since integration provokes deletion of the TYRl coding sequence (tyrosine prototrophy), correct integrants may be easily identified by replica-plating with no need to further verify their chromosomal structure. Finally, the presence of the GAL10 yeast promoter drives the conditional expression of homologous/heterologous genes, allowing easy detection of toxic or lethal effects.

\section{Materials and methods}

\section{Plasmids construction}

The TYR1 locus was obtained by polymerase chain reaction (PCR) of yeast genomic DNA, using oligonucleotides F100 and R100 (for DNA sequences, see Table 1). Both oligonucleotides contain an EcoRV restriction site to facilitate cloning (bold letters in Table 1). G nucleotide was added to both the forward and reverse oligos to create a SalI restriction site necessary for subsequent manipulation (italic letters in Table 1).

Plasmid pTyr was constructed by replacing the 322 bp PvuII fragment of the pUC19 vector, containing the whole polylinker, with the $2023 \mathrm{bp}$ PCR fragment containing the TYRI locus previously digested by EcoRV.

Plasmid pINTyrA was obtained by substituting the 1515 bp StuI fragment of the pTYRI vector with the SUP16 selectable marker.

The GAL10 gene promoter was obtained by two subsequent PCRs. In the first reaction, 100 ng DNA of the YEp51 shuttle vector (Rose and Broach, 1991) was amplified using the oligonucleotides F101 and R101 (for DNA sequence, see Table 1). In the second reaction, $100 \mathrm{ng}$ of the first amplification product was amplified using the oligonucleotides F101 and R101b to insert a polylinker (for DNA sequence, see Table 1). A C nucleotide, non-homologous to the target sequence (for DNA sequence, see Table 1), was added to the F101 oligo to create, after ligation with the StuI-digested pTYRl vector, a Bam $\mathrm{HI}$ site necessary for the subsequent manipulations. Bold letters in Table 1

Table I. List and DNA sequence of oligonucleotides used

\begin{tabular}{ll}
\hline FI00 & 5'-ggcgatatcgTCGACATGGAAAGTGATGTT \\
RI00 & 5'-ggcgatatcgTCGACTITATGACCAAAAACT \\
FIOI & 5'-gcgatatccTCAAAAATCATCGCTTCGC \\
RIOI & 5'-gaattctcgaGCAAAAATCTTACTIIIIITGGA \\
RIOIb & 5'-ggatatcaagcttcccgggGAATTCTCGAGCAAAAA \\
GFP-RT & 5'-TGGGTGCTCAGGTAGTGGTTGTCG \\
GFP-FW & 5'-CCACCTACGGCAAGCTGACCCT \\
GFP-Rev & 5'-TGCCGTCCTCCTTGAATCGAT
\end{tabular}

For all oligonucleotides, capital letters indicate the homologous region, while lower case letters indicate a sequence inserted by the oligonucleotides. Bold letters indicate restriction sites inserted by the oligonucleotide. $g$ in primers FIO0 and RIO0 fused to TCGAC homologous sequence creates a Sall restriction site; $\underline{c}$ in primer FIOI creates a BamHI restriction site after EcoRV digestion and ligation to a TyrL-Stul fragment. 
indicate nucleotides inserted to create an EcoRV restriction site.

The GAL10 gene promoter obtained by the above-mentioned PCRs was TA-cloned onto the PCR2.1 vector (Invitrogen, Carlsbad, CA, USA), generating the PCR2.1GAL vector. Positive colonies were identified by colony PCR and confirmed by restriction analysis and automated DNA sequencing.

Plasmid pINTyr was constructed by substituting the $1515 \mathrm{bp}$ StuI fragment of the pTYRI vector with the 543 bp EcoRV fragment of the PCR2.1GAL vector containing the GAL10 gene promoter. Plasmid PINTyrB was obtained by inserting the 2216 bp LEU2 XhoI/SalI DNA fragment from the Yepl3 vector (Rose and Broach, 1991) within the Bam HI site of the $p G A L T Y R$ vector. The $L E U 2$ fragment and the $p G A L T Y R$ vector were partially filled before ligating in the presence of $\mathrm{C} / \mathrm{T}$ and G/A nucleotides, respectively, to obtain compatible cohesive ends.

Plasmid pINTyrC was obtained inserting the 1355 bp URA3 XbaI DNA fragment from the pUC19URA3 vector within the Bam HI site of the pINTyr vector. The URA3 fragment and the $p G A L$ $T Y R$ vector were partially filled before ligation in the presence of $\mathrm{C} / \mathrm{T} / \mathrm{A}$ and G/T/A nucleotides, respectively, to obtain compatible cohesive ends.

Plasmid pINTyrD was obtained inserting the 772 bp XbaI/SalI DNA fragment from the vector $p E G F P-N 1$ (Clontech Laboratories, Mountain View, USA), containing the complete coding sequence of the GFP gene, within the HindIII/XhoI site of the pINTyrB vector. XbaI and HindIII restriction sites had been previously filled in with the $\mathrm{C} / \mathrm{T}$ and $\mathrm{G} / \mathrm{A}$ nucleotides, respectively.

\section{Yeast relevant genotypes and manipulation}

AAT3B: a ade2 can1-100 CRI4 his3 leu2-3,112 lys $1-1$ ras $1 \dot{\Delta}$ ras $2:: U R A 3$ URA3-52 (Mirisola et al., 1994). F4A2U6: a ade2 can1-100 CRI4 his3 leu2-3,112 lys1-1 ras1 $\dot{\Delta}$ ras $2:: U R A 3$ URA352 (Mirisola et al., 1994). Rich and selective media were as described by Sherman (2002). Standard media with a carbon source different from glucose were obtained by using $2 \%$ galactose in place of glucose. Genetic manipulation of yeasts was as described by Mortimer and Schild (1981). Yeast transformations were carried out as described by Ito et al. (1983).

\section{Yeast genomic DNA extraction}

The yeast strain of interest was incubated on the appropriate medium until saturation (usually $24 \mathrm{~h}$ ). Cells, harvested by centrifugation, were washed twice with water and the pellet, resuspended in 0.05 volumes of lysis buffer [0.1 M Tris-Cl, $\mathrm{pH} 8.0,0.05$ $\mathrm{M}$ ethylenediaminotetracetate (EDTA) $-1 \%$ sodium dodecyl sulphate (SDS)], was added to 0.05 volumes of acid-washed glass beads $(212-300 \mu \mathrm{m}$ mesh; Sigma-Aldrich, Milan, Italy). The mixture was vortexed for $30 \mathrm{~s}$ and $\mathrm{NaCl}$ was added to a final concentration of $0.250 \mathrm{M}$. After vortexing, the phases were separated by centrifugation at $9000 \times g$ for $8 \mathrm{~min}$. The aqueous phase was phenol : chloroform-extracted and the resulting supernatant was ethanol-precipitated. After a $70 \%$ ethanol wash, the pellet was finally resuspended in $20 \mu \mathrm{l}$ water. Genomic DNA was further purified using a commercial kit (GFX PCR DNA and gel band purification, GE Healthcare, Little Chalfont, UK) before being subjected to further manipulations.

\section{RNA preparation}

The desired yeast strain was grown at $30^{\circ} \mathrm{C}$ on the appropriate selective glucose-based medium to $\mathrm{OD}_{560 \mathrm{~nm}}=0.8 / 1$. The cells were collected by centrifugation and resuspended in the same medium, except for induced cultures, in which galactose replaced glucose at the same concentration and which were cultured at $30^{\circ} \mathrm{C}$ for an additional $24 \mathrm{~h}$. After centrifugation, the pellet, resuspended in acetate buffer $(50 \mathrm{~mm}$ sodium acetate, $10 \mathrm{~mm}$ EDTA, $\mathrm{pH} 5$ ), was vortexed for $5 \mathrm{~min}$ at $65^{\circ} \mathrm{C}$ in the presence of one volume of acid-washed glass beads (212-300 $\mu \mathrm{m}$ mesh) and one volume of phenol, $\mathrm{pH}$. The aqueous phase was extracted twice with chloroform and RNA was finally precipitated with 2.5 volumes of absolute ethanol, $0.3 \mathrm{M}$ sodium acetate, $\mathrm{pH}$ 5.5. After centrifugation, the pellet was resuspended in $10 \mathrm{~mm}$ Tris. C1, $1 \mathrm{~mm}$ EDTA, pH8 (TE $10: 1)$. The RNA quality was verified by evaluating the ribosomal RNA appearance after gel electrophoresis in denaturing conditions. Poly $(\mathrm{A})^{+}$ RNA was obtained using a commercially available kit (QuickPrep ${ }^{\mathrm{TM}}$ Micro mRNA Purification Kit, GE Healthcare), according to the manufacturer's instructions. 


\section{RT-PCR}

$1 \mu \mathrm{g} \operatorname{poly}(\mathrm{A})^{+}$RNA was subjected to GFP-specific retrotranscription (RT) with the GFP-RT primer (for DNA sequence, see Table 1) in the presence of $1 \mu \mathrm{l}$ Improm reverse transcriptase (Promega Corp., Madison, WI, USA) at $42{ }^{\circ} \mathrm{C}$ for $1 \mathrm{~h}$, according to the manufacturer's instructions. Different amounts of cDNA were used for the subsequent PCR. PCR of cDNA was performed using the GFP-Fw and GFP-Rev primers indicated in Table 1 under the following conditions: $94^{\circ} \mathrm{C}$ for $5 \mathrm{~min}$, followed by 30 cycles of: $94^{\circ} \mathrm{C}$ for $30 \mathrm{~s} ; 58^{\circ} \mathrm{C}$ for $30 \mathrm{~s} ; 72^{\circ} \mathrm{C}$ for $1 \mathrm{~s}$.

\section{Results and discussion}

\section{Disrupting the TYRI locus: resulting phenotype}

As a first step, we engineered a disruption vector targeted to the TYRl locus. The chromosomal TYRl gene was obtained by PCR cloning (for details, see above and Table 1). The TYR1 amplicon contained a SalI restriction site at both extremities, created by adding, during PCR, a G nucleotide to a TCGAC sequence occurring within the natural TYRI sequence (see primers F100 and R100 of Table 1). The amplicon was cloned onto a modified Escherichia coli pUC19 vector lacking the whole polylinker to generate the $p T Y R 1$ vector (not shown). After cloning, the described Sal I restriction sites were used to recover the TYRI DNA fragment (recombination cassette), leaving at both extremities only naturally occurring, and thus highly recombinogenic, TYRl sequences. In order to use this construct for targeted disruption of the TYRI locus, the vector's StuI fragment, containing the whole coding sequence of the cloned TYR1, was substituted with the SUP16 selectable marker to generate the pINTyrA vector shown in Figure 1. This disruption vector contained two small (278 and 234 bp) TYRl homologous regions (respectively TL and TR in Figure 1) surrounding a selectable marker (SUP16) and was capable of directing ends-out gene disruption of the TYRI locus.

To confirm the previously identified tyrosine prototrophy subsequent to TYRI mutations (Mannhaupt et al., 1989) using our pINTyr A disruption vector, competent cells of the yeast strains
AAT3B and F4A2U6 (with the same relevant genotype but obtained by different crossings; Mirisola et al., 1994) were transformed using the purified INTyrA recombination cassette (see Figure 1) obtained after SalI restriction of the pINTyr A vector and agarose gel purification of the fragment. Genomic DNA extracted from selected transformants were analysed to check the chromosomal structure of the TYRI locus. A mixture of 10 individual clones with the correct chromosomal structure of both strains were replica-plated onto the same medium but lacking tyrosine. As shown in Figure 2, TYRl deletion provokes tyrosine prototrophy in both yeast strains, confirming the possibility of using TYRl as a selectable marker. The recombinant strains were also assayed in different growth conditions and in the presence of different carbon sources without showing any additional phenotype (results not shown).

\section{Construction of the integrative/expression vector and verification}

We took advantage of the above-demonstrated property of the pINTyr A vector integration to display two independent phenotypes to create an easyto-screen family of integration vectors for general usage. The StuI fragment of the pTYR1 vector, described above, was exchanged with the EcoRV fragment containing the GAL10 gene promoter, the latter obtained by two rounds of PCR (see above). Oligonucleotides used for PCR introduced a polylinker at the $3^{\prime}$ end of the GAL1O gene promoter and, after ligation to the vector, created a unique Bam $\mathrm{HI}$ restriction site at the $5^{\prime}$ end of the promoter (pINTyr vector, not shown). LEU2 or URA3 markers were inserted as a XhoI/SalI DNA fragment from the YEp13 vector and $X b a \mathrm{I}$ DNA fragment from the $p U C 19 U R A 3$ vector, respectively, and ligated, after partial fill, to the $B a m \mathrm{HI}$ restriction site to produce pINTyrB and pINTyrC vectors, respectively (for details, see above and Figure 1). After SalI restriction, the gel-purified recombination cassette from $p I N T y r B$ and $p I N$ Tyr C vectors were used to transform yeast competent cells of the AAT3B strain. After selection for leucine or uracil auxotrophy, depending on the vector used, transformed cells were replicaplated on a medium lacking tyrosine. In our experiments all clones showing tyrosine prototrophy had the correct chromosomal structure, demonstrating 


\section{Available recombination cassettes $\quad$ Effect upon integration}
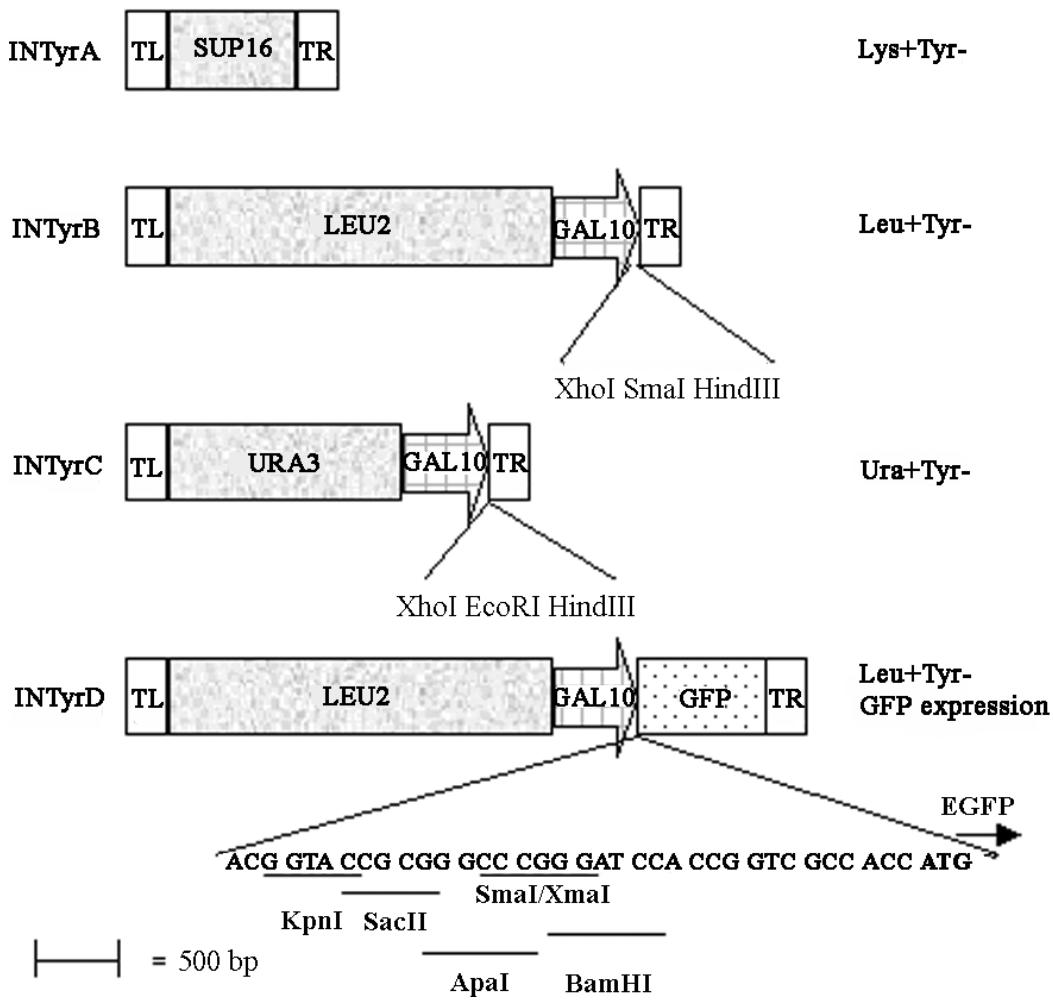

Figure I. List of all recombination cassettes available. Each recombination cassette may be obtained from the corresponding circular vector by Sall restriction and agarose gel purification

that this vector, thanks to the double selection it drives, facilitates the identification of transformed yeast cells with the desired chromosomal structure.

To test the conditional expression of sequences cloned within our integrative vector, the XbaI-SalI fragment of the $p E G F P-N 1$ vector, containing the complete coding sequence of the green fluorescent protein (GFP) gene, was cloned within the HindIII-XhoI site of the pINTyrB vector, generating the pINTyrD vector (see Figure 1). After chromosomal integration of the construct, recombinant yeast cells were grown on a glucose-based medium and an aliquot of the culture was transferred to a galactose-based medium to induce the GAL10-driven expression of GFP. Total RNA was extracted from both glucose- and galactosegrown cells and subjected to RT-PCR, using oligonucleotides directed against the GFP coding sequence (see Materials and methods). The results, shown in Figure 3, confirm the conditional expression of the GFP cloned in our integrative vector. It is worth noting that pINTyrD vector may be used to generate SOI-GFP fusion proteins and to monitor their subcellular compartmentalization.

We therefore conclude that these vectors can improve the ends-out integrative procedure of DNA sequences onto the yeast genome. The presence of a yeast promoter within the recombination cassette allows expression of sequences from every organism. Furthermore, the possibility of conditionally expressing the SOI sequence can be helpful in cases where a toxic or lethal effect is possible. In addition, since the TYRI locus is not subjected to chromatin silencing by the known deacetylases Hda1 and Sir2 (Robyr et al., 2002), this vector may be useful to free yeast gene expression from this control level. The double selection based on tyrosine prototrophy and leucine or uracil auxotrophy 
+ Tyrosine

Tyr1::SUP16 TYR1 wt

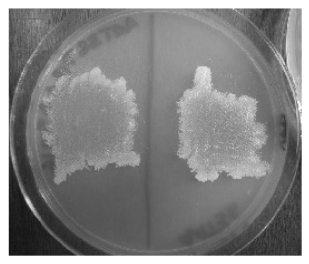

AAT3B

F4A2U6

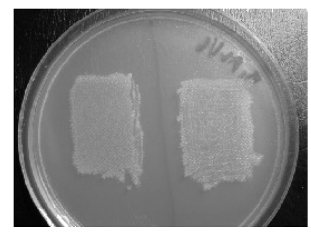

- Tyrosine

Tyr1::SUP16 TYR1 wt
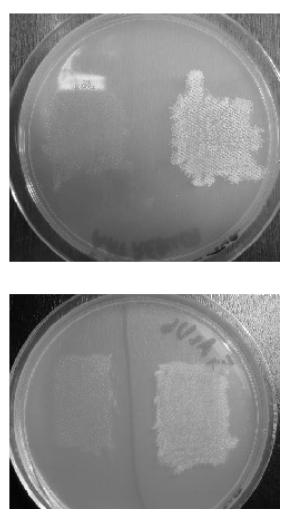

Figure 2. Growth properties of strains with TYRI deletion. Yeast competent cells of two different yeast strains (AAT3B and F4A2U6) were transfected with $10 \mu \mathrm{g}$ INTyrA recombination cassette (TYRI::SUPI6) or with the corresponding empty vector (TYRI wt). A pool of 10 selected integrants with the correct chromosomal structure were grown on complete medium ( + tyrosine) and replica-plated on a medium lacking tyrosine ( - tyrosine). The plates were photographed after $48 \mathrm{~h}$ growth at $30^{\circ} \mathrm{C}$

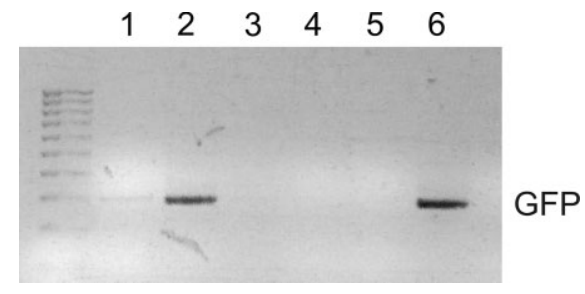

Figure 3. GFP-specific RT-PCR of RNA extracted from different yeast strains grown in different conditions. Total RNA from the strain AAT3B TYRI::INTyrD $(I-4)$ grown on glucose $(I, 3)$ or galactose $(2,4)$, and from the parental strain AAT3B grown on galactose (5), were subjected to RT-PCR $(I, 2,5)$ or used dierctly $(3,4)$ for PCR, using GFP-specific oligonucleotides. PCR using pINTyrD plasmid DNA as a template (6) was used as a positive control

guarantees an easy identification of correct integrants. It must be noted that, since the recombination cassette can only be released by SalI restriction digestion, the SalI restriction site must be absent within the SOI. Finally, the pINTyr vector may be used to clone, within the unique Bam HI restriction site, selectable markers different from those used in the present work.

\section{References}

Bowers K, Stevens TH. 2005. Protein transport from the late Golgi to the vacuole in the yeast Saccharomyces cerevisiae. Biochim Biophys Acta 10(3): 438-454.

Coughlan CM, Brodsky JL. 2005. Use of yeast as a model system to investigate protein conformational diseases. Mol Biotechnol 30(2): 171-180.

Hartwell LH. 2002. Nobel Lecture. Yeast and cancer. Biosci Rep 22(3-4): 373-394.

Hastings PJ, McGill C, Shafer B, Strathern JN. 1993. Ends-in vs. ends-out recombination in yeast. Genetics 135: 973-980.

Ito H, Fukuda Y, Murata K, Kimura A. 1983. Transformation of intact yeast cells treated with alkali cations. J Bacteriol 153(1): $163-168$.

Kaeberlein M, McVey M, Guarente L. 2001. Using yeast to discover the fountain of youth. Sci Ageing Knowl Environ 1: 1 [review].

Langston LD, Symington LS. 2004. Gene targeting in yeast is initiated by two independent strand invasions. Proc Natl Acad Sci USA 101: 15392-15397.

Lindquist SL. 2002. Prion proteins: one surprise after another. Harvey Lect 98: 173-205.

Longo VD. 2004. Ras: the other pro-ageing pathway. Sci Ageing Knowl Environ 29(39): 36.

Mannhaupt G, Stucka R, Pilz U, Schwarzlose C, Feldmann H. 1989. Characterization of the prephenate dehydrogenaseencoding gene, TYRl, from Saccharomyces cerevisiae. Gene $\mathbf{8 5}$ : 303-311.

Mirisola MG, Seidita G, Verrotti AC, Di Blasi F, Fasano O. 1994. Mutagenic alteration of the distal switch II region of RAS blocks CDC25-dependent signaling functions. J Biol Chem 269(22): 15740-15748.

Mortimer R, Schild D. 1981. The Molecular Biology of the Yeast Saccharomyces cerevisiae: Life Cycle and Inheritance, Strathern JL, Jones EW, Broach JR (eds). Cold Spring Harbor Laboratory Press: Cold Spring Harbor, NY; 11-26.

Nakano A. 2004. Yeast Golgi apparatus - dynamics and sorting. Cell Mol Life Sci 61(2): 186-191.

Robyr D, Suka Y, Xenarios I, et al. 2002. Microarray deacetylation maps determine genome-wide functions for yeast histone deacetylases. Cell 109: 437-446.

Rose MD, Broach JR. 1991. Cloning genes by complementation in yeast. Methods Enzymol 194: 195-230.

Schneider JC, Guarente L. 1991. Vectors for expression of cloned genes in yeast: regulation, overproduction and underproduction. Methods Enzymol 194: 373-388.

Sherman F. 2002. Getting started with yeast. Methods Enzymol 350: $3-41$.

Sherman MY, Muchowski PJ. 2003. Making yeast tremble: yeast models as tools to study neurodegenerative disorders. Neuromol Med 4(1-2): 133-46.

Tissenbaum HA, Guarente L. 2002. Model organisms as a guide to mammalian ageing. Dev Cell 2: 9-19 [review].

Voth WP, Richards JD, Shaw JM, Stillman DJ. 2001. Yeast vectors for integration at the $\mathrm{HO}$ locus. Nucleic Acids Res 29(12): E59. 